\title{
Low bend loss waveguides and photonic bandgaps enabled by high index contrast modifications
}

\author{
Simon Gross, ${ }^{1,2 *}$ Alexander Arriola, ${ }^{1,2,3}$ Nemanja Jovanovic, ${ }^{1,4,5}$ \\ Ned Charles, ${ }^{6}$ Peter G. Tuthill, ${ }^{6}$ Santiago M. Olaizola, ${ }^{3}$ \\ Alexander Fuerbach, ${ }^{1,2}$ and Michael J. Withford ${ }^{1,2,4}$ \\ ${ }^{1}$ MQ Photonics Research Centre, Dept. of Physics and Astronomy, Macquarie University, NSW 2109, Australia \\ ${ }^{2}$ Centre for Ultrahigh-bandwidth Devices for Optical Systems (CUDOS), Australia \\ ${ }^{3}$ CEIT and Tecnun, University of Navarra, $P^{\circ}$ Manuel de Lardizabal 15,Donostia-San Sebastian, 20018, Spain \\ ${ }^{4}$ Macquarie University Research Centre in Astronomy, Astrophysics and Astrophotonics, Dept. of Physics and Astronomy, Macquarie University, \\ NSW 2109, Australia \\ ${ }^{5}$ Australian Astronomical Observatory (AAO), PO Box 915, North Ryde NSW 1670, Australia \\ ${ }^{6}$ Sydney Institute for Astronomy (SIFA), School of Physics, University of Sydney, 2006, Australia \\ simon.gross@mq.edu.au
}

\begin{abstract}
We present a novel process to fabricate high index contrast waveguides in boroaluminosilicate glass based on differential thermal post-annealing. Firstly, large high index contrast multimode waveguides are inscribed and subsequently annealed to achieve single-mode operation. We applied this technique to significantly increase the throughput of 3D astrophotonic devices and to demonstrate anti-resonant (ARROW) guiding in a laser written structure for the first time.
\end{abstract}

\section{Introduction}

Since the first demonstration of femtosecond laser written waveguides in 1996, the femtosecond direct-write technique has found applications in a variety of fields, like astrophotonics, quantum photonics and microfluidics [14]. The interaction between the glass and tightly focused femtosecond beam strongly depends on the laser's repetition rate. Using low repetition rate pulses, where the time between successive pulses is significantly larger than the thermal diffusion time of glass $(\sim 1 \mu \mathrm{s})$ leads to a smooth repetitive modification of the glass with a Gaussian refractive index profile, that reflects the shape of the laser's intensity profile. However for providing sufficient spatial pulse-to-pulse overlap slow translation speeds have to be chosen where fabrication of a single device can take several hours. In contrast, using high repetition rates shortens the fabrication time to a few minutes. However the high repetition rate pulse train results in local melting following by rapid quenching of the glass. In common multicomponent silicate glasses this generally creates a complex refractive index profile. It has been shown, that the bend losses of such an index profile are significantly larger than these for a step-index profile with similar modefield diameter [5]. To overcome this shortcoming we developed a two-step fabrication process where firstly, a large multimode waveguide is inscribed as shown in Fig. 1a.
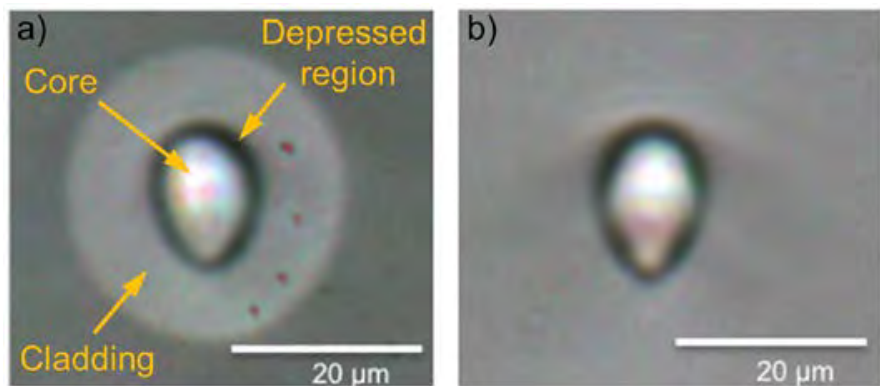

Fig. 1: a) Optical microscope image of the waveguide cross section before annealing in Corning Eagle 2000 boro-aluminosilicate glass. b) Waveguide after annealing.

The waveguide cross section consist of three distinct regions, a high index contrast core which is surrounded by a region of depressed refractive index and a cladding of raised index. The second process step exploits the different thermal stabilities of those regions, where the glass is heated above its annealing point and slowly cooled back down to room temperature. This results in erasure of the cladding but preserves the high index core and depressed region and thereby creating a single-mode waveguide with a peak index contrast of $\sim 7 \times 10^{-3}$ as shown in Fig. $2 \mathrm{~b}$. 


\section{Application to astrophotonics}

We have successfully applied this fabrication process to significantly increase the throughputs of a 3D astrophotonic device known as a 'pupil-remapper' (details in [6]). The chip contains 8 pathlength-matched waveguides with bend radii of 23 to $35 \mathrm{~mm}$ to remap the light from a 2D input plane into a linear array at the output. While previously the raw throughputs for the device ranged between $5 \%$ and $47 \%$, the differential thermal annealing significantly increased the fidelity of the device with constant high throughputs of 70-73\% across all 8 waveguides (Fig. 2a).
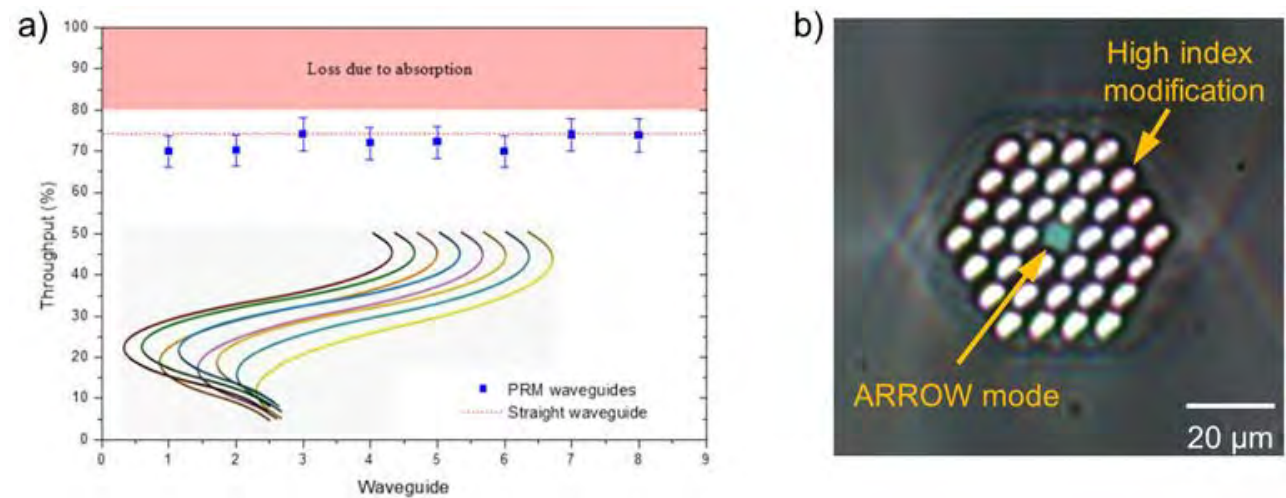

Fig. 2: a) Measured throughputs of the pupil-remapper chip. The inset shows an artists' impression of the device. b) Anti-resonant guiding in a hexagonal lattice of positive refractive index modifications under white light illumination.

\section{Application to anti-resonant reflecting optical waveguides}

An anti-resonant reflecting optical waveguide (ARROW) is a waveguide were a low index core is surrounded by several high index layers/inclusions. Guiding in such a structure is governed by light either leaking out of the core if being in resonance with the high index inclusions or reflecting back in the case of anti-resonance [7]. This resonant behaviour results in photonic bandgaps where each bandgap possess a zero-dispersion wavelength. ARROW fibres have been demonstrated with index contrasts down to $1 \times 10^{-2}$ [8]. However attempts to fs laser inscribe such a structure have failed due to limited index contrast until now [9]. A fs laser inscribed ARROW waveguide is shown in Fig. 2b consisting of a hexagonal lattice of high index modification with a defect in the centre to form a core. These waveguides provide an avenue to dispersion engineering of femtosecond laser written devices for the first time.

\section{References}

1. R. R. Gattass and E. Mazur, "Femtosecond laser micromachining in transparent materials," Nature Photonics 2, $219-225$ (2008).

2. R. R. Thomson, A. K. Kar, and J. Allington-Smith, "Ultrafast laser inscription: an enabling technology for astrophotonics," Optics Express 17, 1963 (2009).

3. G. D. Marshall, A. Politi, J. C. F. Matthews, P. Dekker, M. Ams, M. J. Withford, and J. L. O’Brien, "Laser written waveguide photonic quantum circuits.," Optics Express 17, 12546-54 (2009).

4. R. Osellame, H. J. W. M. Hoekstra, G. Cerullo, and M. Pollnau, "Femtosecond laser microstructuring: an enabling tool for optofluidic labon-chips," Laser \& Photonics Reviews 5, 442-463 (2011).

5. N. Charles, N. Jovanovic, S. Gross, P. Stewart, B. Norris, J. O’Byrne, J. S. Lawrence, M. J. Withford, and P. G. Tuthill, "Design of optically path-length-matched, three-dimensional photonic circuits comprising uniquely routed waveguides," Applied Optics 51, 6489 (2012).

6. N. Jovanovic, P. G. Tuthill, B. Norris, S. Gross, P. Stewart, N. Charles, S. Lacour, M. Ams, J. S. Lawrence, a. Lehmann, C. Niel, J. G. Robertson, G. D. Marshall, M. Ireland, a. Fuerbach, and M. J. Withford, "Starlight demonstration of the Dragonfly instrument: an integrated photonic pupil-remapping interferometer for high-contrast imaging," Monthly Notices of the Royal Astronomical Society 427, 806-815 (2012).

7. N. M. Litchinitser, a K. Abeeluck, C. Headley, and B. J. Eggleton, "Antiresonant reflecting photonic crystal optical waveguides," Optics Letters 27, 1592 (2002).

8. A. Argyros, T. A. Birks, S. G. Leon-Saval, C. M. B. Cordeiro, F. Luan, and P. S. J. Russell, "Photonic bandgap with an index step of one percent," Optics Express 13, 309 (2005).

9. A. Fuerbach, S. Gross, M. Alberich, D. Lancaster, H. Ebendorff-Heidepriem, T. Monro, and M. Withford, "Femtosecond laser direct-written microstructured waveguides in passive as well as in novel active glasses," 2012 14th International Conference on Transparent Optical Networks (ICTON) 1-4 (2012). 